\title{
DUAL-DEPTH ADAPTED IRREDUCIBLE FORMAL MULTIZETA VALUES
}

\author{
LEILA SCHNEPS
}

\begin{abstract}
Let $\delta \mathfrak{s}$ denote the double shuffle Lie algebra, equipped with the standard weight grading and depth filtration; we write $\mathfrak{D S}=\oplus_{n \geq 3} \delta \mathfrak{S}_{n}$ and denote the filtration by $\delta \mathfrak{S}^{1} \supset \delta \mathfrak{S}^{2} \supset \ldots$. The double shuffle Lie algebra is dual to the new formal multizeta space $\mathfrak{n} \mathfrak{f} z=\oplus_{n \geq 3} \mathfrak{n} \mathfrak{f} z_{n}$, which is equipped with the dual depth filtration $\mathfrak{n} \tilde{f}_{z}^{1} \subset \mathfrak{n} \tilde{f}^{2} \subset \ldots$ Via an explicit canonical isomorphism $\boldsymbol{D} \mathfrak{g} \stackrel{\sim}{\rightarrow} \mathfrak{n} \tilde{f} z$, we define the "dual" in $n \mathfrak{n} z$ of an element in $\delta \mathfrak{s}$. For each weight $n \geq 3$ and depth $d \geq 1$, we then define the vector subspace $\mathfrak{D} \mathfrak{S}_{n, d}$ of $\mathfrak{D} \mathfrak{s}$ as the space of elements in $\mathfrak{D} \mathfrak{S}_{n}^{d}-\mathfrak{D} \mathfrak{S}_{n}^{d+1}$ whose duals lie in $\mathfrak{n} \mathfrak{f}_{n}^{d}$. We prove the direct sum decomposition

$$
\mathfrak{D} \mathfrak{S}=\bigoplus_{n \geq 3} \bigoplus_{d \geq 1} \mathfrak{D}_{\mathfrak{S}_{n, d}},
$$

which yields a canonical vector space isomorphism between $\mathfrak{D} \mathfrak{g}$ and its associated graded for the depth filtration, $\mathfrak{D} \mathfrak{S}_{n, d} \simeq \mathfrak{D} \mathfrak{S}_{n}^{d} / \mathfrak{D} \mathfrak{S}_{n}^{d+1}$. A basis of $\mathfrak{D} \mathfrak{s}$ respecting this decomposition is dual-depth adapted, which means that it is adapted to the depth filtration on $\mathrm{D} \mathfrak{s}$, and the basis of dual elements is adapted to the dual depth filtration on $\mathfrak{n} \mathfrak{f}_{z}$. We use this notion to give a canonical depth 1 generator $f_{n}$ for $\mathrm{D} s$ in each odd weight $n \geq 3$, namely the dual of the new formal single zeta value $\zeta(n) \in \mathfrak{n} \tilde{f}_{z}$. At the end, we also apply the result to give canonical irreducibles for the formal multizeta algebra in weights up to 12 .
\end{abstract}

\section{Formal multizeta values and the double shuffle}

We begin by briefly recalling the definitions of the Hopf algebra of formal multizeta values, the double shuffle Lie algebra, their duals, and the relations between them.

Consider the following diagram, in which the four top spaces are Hopf algebras. The top right-hand space is the free polynomial ring in two noncommutative variables, and the top left-hand space is its dual, with the dual of a word $w \in \mathrm{Q}\langle x, y\rangle$ denoted by the formal symbol $\bar{Z}(w)$. The space $\mathrm{Q}\langle x, y\rangle$ is equipped with the standard coproduct defined by $\Delta(x)=x \otimes 1+1 \otimes x$ and $\Delta(y)=y \otimes 1+1 \otimes y$, and its dual is equipped with the Goncharov coproduct $\Delta_{G}$ (cf. [4]). The multiplication on $\mathrm{Q}[\bar{Z}(w)]$ is the shuffle; the multiplication on $\mathrm{Q}\langle x, y\rangle$ is difficult to write down explicitly, but is defined by being dual

Received 24 June, in final form 4 November 2011. 
to $\Delta_{G}$. The top horizontal arrow simply maps a word $w$ to the formal dual symbol $\bar{Z}(w)$.

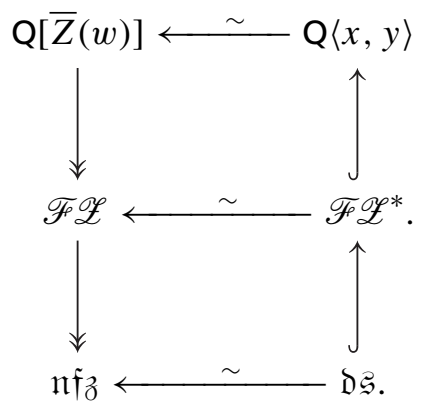

The Hopf algebra of formal multizeta values $\mathscr{F} \mathscr{Z}$ is the quotient of $\mathrm{Q}[\bar{Z}(w)]$ modulo the regularized shuffle and stuffle relations; we write $Z(w)$ for the image of $\bar{Z}(w)$ in this space.

All spaces in the diagram inherit the positive weight grading from the top level, which is simply the degree of monomials in $x$ and $y$. On the left-hand side of the diagram, this follows from the fact that the linear relations defining the quotients all take place within fixed weights. In particular, the weight 0 part of the top four spaces is equal to Q.

At the bottom left is the Lie coalgebra of new formal multizeta values, obtained by quotienting $\mathscr{F} \mathscr{Z}$ by the ideal generated by $\mathscr{F} \mathscr{Z} 0=\mathrm{Q}$, by $Z(x y)$ (usually denoted $Z(2)$ under the frequently used notation $Z\left(x^{k_{1}-1} y \ldots x^{k_{r}-1} y\right)=$ $Z\left(k_{1}, \ldots, k_{r}\right)$ for words $w$ ending in $\left.y\right)$, and by products $(\mathscr{F} \mathscr{Z} \geq 1)^{2}$. The Goncharov coproduct passes to a Lie cobracket $\delta_{G}$ on $\mathfrak{n} \mathfrak{f} z$ (by first removing the primitive part, i.e. setting $\Delta_{G}^{\prime}(x)=\Delta_{G}(x)-x \otimes 1-1 \otimes x$, and then taking $\delta_{G}$ to be $\Delta_{G}^{\prime}$ modulo products). Any vector space basis of $\mathfrak{n} \mathfrak{f} z$, together with 1 and $Z(2)$, yields a system of multiplicative generators ("irreducibles") for the Q-algebra $\mathscr{F} \mathscr{Z}$. One of the frequently asked questions about $\mathscr{F} \mathscr{Z}$ is whether there exists a canonical, or at least a very natural system of irreducibles.

The dual of $\mathfrak{n} \mathfrak{f} z$ is known as the double shuffle Lie algebra $\mathfrak{D} \mathfrak{s}$. The horizontal arrows in the diagram are duality isomorphisms obtained by restricting the top arrow $w \mapsto \bar{Z}(w)$ to the lower right-hand entries of the diagram, which are subsets of $\mathrm{Q}\langle x, y\rangle$.

The space $\mathrm{Q}\langle x, y\rangle$ is equipped with a depth filtration defined by taking the depth of a polynomial $f(x, y)$ to be the smallest number of $y$ 's occurring in any monomial of $f$; the space $\mathrm{Q}^{d}\langle x, y\rangle$ is the vector space of polynomials of depth $\geq d$. This is a decreasing filtration $\mathrm{Q}^{d}\langle x, y\rangle \supset \mathrm{Q}^{d+1}\langle x, y\rangle$, and by intersection it induces a decreasing filtration on the subspaces $\mathscr{F}^{2} \mathscr{Z}^{*}$ and $\mathfrak{n} \mathfrak{f}^{*}=\mathfrak{d} \mathfrak{s}$. Each of the left-hand spaces $\mathscr{A}$ is equipped with the dual filtration, in which on each horizontal level $\mathscr{A} \leftarrow \mathscr{A}^{*}$, we define $\mathscr{A}^{d}$ to be the subspace 
of $\mathscr{A}$ annihilated by $\left(\mathscr{A}^{*}\right)^{d+1}$; thus the filtration on the left-hand spaces is increasing, $\mathscr{A}^{d} \subset \mathscr{A}^{d+1}$. The filtrations induce filtrations on each weight graded piece $\mathscr{A}_{n}$ and $\mathscr{A}_{n}^{*}$. By duality, on each horizontal level $\mathscr{A} \leftarrow \mathscr{A}^{*}$ of the diagram we have

$$
\left(\mathscr{A}^{*}\right)_{n}^{d} /\left(\mathscr{A}^{*}\right)_{n}^{d+1} \simeq \mathscr{A}_{n}^{d} / \mathscr{A}_{n}^{d-1}
$$

One can also give a direct definition of the double shuffle Lie algebra by characterizing the polynomials of $\mathrm{Q}\langle x, y\rangle$ which lie in $\mathfrak{D} \mathfrak{S}$ as follows.

Let Lie $[x, y]$ denote the free Lie algebra on two generators, with a weight grading given by polynomial degree. For a polynomial $f$ in $x$ and $y$ and any word $w$ in $x$ and $y$, let $(f \mid w)$ denote the coefficient of the word $w$ in $f$, and extend this definition to $(f \mid g)$ for polynomials $g$ in $x$ and $y$ by right linearity. Then the underlying vector space of the double shuffle Lie algebra $D \mathfrak{s}$ can be defined as follows:

$$
\mathfrak{D} \mathfrak{S}=\left\{f \in \operatorname{Lie}_{\geq 3}[x, y] \mid(f \mid s t(u, v))=0 \forall u, v \in \mathbf{Q}\langle x, y\rangle y\right\},
$$

where $s t(u, v)$ denotes the stuffle product of words ending in $y$. For every $f \in \operatorname{Lie}[x, y]$, let $D_{f}$ be the associated derivation of $\operatorname{Lie}[x, y]$ defined by $D_{f}(x)=0, D_{g}(y)=[y, f]$. The Poisson bracket

$$
\{f, g\}=[f, g]+D_{f}(g)-D_{g}(f)
$$

arises naturally from bracketing derivations since $\left[D_{f}, D_{g}\right]=D_{f} \circ D_{g}-$ $D_{g} \circ D_{f}=D_{\{f, g\}}$. One of the main known results concerning $\mathfrak{D}_{\mathfrak{g}}$ is that it is actually a Lie algebra under the Poisson bracket. There are two independent and very different proofs of this fact. Racinet [5] gave a direct proof (but see the appendix of [3] for a simplified exposition). Ecalle [1] gave a very different proof, by embedding the whole situation in the vaster "ARI/GARI" theory.

\section{Canonical dual-depth adapted decomposition of the double shuffle Lie algebra}

In this section we show how the double shuffle Lie algebra $D$ s is canonically isomorphic, as a vector space, to its associated graded $\oplus \mathcal{D} \mathfrak{S}_{n}^{d} / \mathfrak{D} \mathfrak{S}_{n}^{d+1}$, and how this leads to the choice of particularly well-adapted bases for the depth filtrations of $\mathfrak{b} \mathfrak{s}$ and its dual $\mathfrak{n} \mathfrak{f} z$.

The striking fact about placing the Lie algebra $D S$ and its new formal multizeta value dual $\mathfrak{n} \mathfrak{f} z$ within the diagram (1) is that the canonical top arrow $\delta: \mathrm{Q}\langle x, y\rangle \stackrel{\sim}{\rightarrow} \mathrm{Q}[\bar{Z}(w)]$ induces explicit canonical duality isomorphisms (also called $\delta$ ) all the way down the diagram, i.e. isomorphisms

$$
\delta: \mathscr{F} \mathscr{Z}^{*} \stackrel{\sim}{\rightarrow} \mathscr{F} \mathscr{Z}, \quad \delta: \mathfrak{D} \mathfrak{g} \stackrel{\sim}{\rightarrow} \mathfrak{n} \mathfrak{i} z .
$$


The maps $\delta$ are completely explicit: to calculate the image of a polynomial $f$ under $\delta$, one first replaces each word $w$ in $f$ by the formal symbol $\bar{Z}(w)$, then replaces each non-convergent symbol $\bar{Z}(w)$ by a linear combination of convergent symbols ("shuffle regularization") using for example the explicit formulas in Prop. 3.2.3 of [2], and finally passes to the quotient modulo the linear system of regularized stuffle relations.

For odd $n \geq 3$, let $z(n) \in \mathfrak{n} \mathfrak{f} z$ denote the image in $\mathfrak{n} \mathfrak{f} z$ of the single zeta value $Z(n) \in \mathscr{F} \mathscr{Z}$. The intuition which makes it desirable to single out elements of DS which are "dual to" the new single zeta values $z(n) \in \mathfrak{n} \mathfrak{f} z$ for odd $n \geq 3$ acquires a meaning in this context; there is a "dual element" of $z(n)$ in $\mathfrak{D} \mathfrak{S}$, namely the unique depth 1 element $f_{n} \in \mathcal{D} \mathfrak{S}_{n}$ which maps to $z(n)$ under the duality isomorphism $\delta$ of (3).

Definition 2.1. A canonical system of depth 1 generators in odd weights $n \geq 3$ for $\mathrm{D} s$ is given by the set of elements

$$
f_{n} \in \mathfrak{D} \mathfrak{s}_{n} \quad \text { such that } \quad \delta\left(f_{n}\right)=z(n) \in \mathfrak{n} \mathfrak{f}_{\mathfrak{z}_{n}} .
$$

In the following theorem, we generalize this approach to finding "good" bases of the double shuffle Lie algebra, and in $\S 3$, we will use the map $\delta$ to turn these bases into systems of ring generators for $\mathscr{F} \mathscr{Z}$.

THEOREM 2.2.

(i) The Lie algebra Ds has a canonical decomposition as a direct sum of subvector spaces

$$
\mathfrak{D} \mathfrak{s}=\bigoplus_{n \geq 3} \bigoplus_{d \geq 1} \mathfrak{D}_{\mathfrak{s}} n, d,
$$

where $\mathfrak{D} \mathfrak{S}_{n, d}=\left\{f \in \mathfrak{D} \mathfrak{S}_{n}^{d}-\mathfrak{D} \mathfrak{S}_{n}^{d+1} \mid \delta(f) \in \mathfrak{n} \mathfrak{f}_{n}^{d}\right\}$.

(ii) For every $n \geq 3, d \geq 1$, the map

$$
\mathfrak{D} \mathfrak{S}_{n, d} \rightarrow \mathfrak{D} \mathfrak{S}_{n}^{d} / \mathfrak{D} \mathfrak{S}_{n}^{d+1}
$$

sending an element of $\mathrm{D}_{\mathfrak{S}_{n, d}} \subset \mathrm{D} \mathfrak{S}_{n}^{d}$ to the quotient is an isomorphism. Thus, as a vector space, DS is canonically isomorphic to its associated graded for the depth filtration.

REMARK 2.3. The existence of a canonical system of irreducibles (ring generators) for $\mathscr{F} \mathscr{Z}$ has been an open question for a long time. The present paper does not give a unique system of irreducibles, but it gives rise to systems having the agreeable property of being dual-depth adapted. To obtain a set of irreducibles for $\mathscr{F} \mathscr{Z}$ from a basis of $D \mathfrak{S}$, one considers the basis elements 
as lying in $\mathscr{F}_{\mathscr{Z}^{*}}$, and takes their duals (images under $\delta$ ) in $\mathscr{F} \mathscr{Z}$. Note that in fact, the choice of irreducible is canonical up to scalar multiple whenever $\operatorname{dim} D \mathfrak{S}_{n, d}=1$; this is always the case for $d=1$ and odd $n$, as we saw (the spaces $\delta \mathfrak{s}_{n, 1}=0$ for even $n$ ), and also for all $d$ in weights up to 12 . We can thus give a complete table of canonical irreducibles for $\mathscr{F} \mathscr{Z}$ up through weight 12 , which is done in $\S 3$.

REMARK 2.4. The decomposition (4) of DS does not respect the Lie algebra structure of $D \mathfrak{s}$, in the sense that

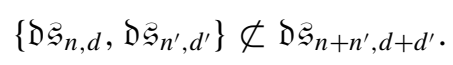

Indeed, take $f_{3} \in \mathcal{D}_{3}{ }_{3}^{1}, f_{9} \in \mathcal{D} \mathfrak{S}_{9}^{1}$; then the dual of the Poisson bracket $\left\{f_{3}, f_{9}\right\}$ lies in $\mathfrak{n} \tilde{f}^{4}{ }_{12}^{4}$ but not in $\mathfrak{n} \mathfrak{f}_{z_{12}}^{2}$.

The proof of Theorem 2.2 is just a consequence of totally general results on filtered vector spaces and their filtered duals, when these vector spaces are linked by an explicit isomorphism mapping. Let us first explain the general situation.

Definition 2.5. Let $V$ be a finite-dimensional vector space equipped with an increasing (resp. decreasing) filtration and a fixed isomorphism $\delta: V^{*} \rightarrow$ $V$. A basis for $V$ is said to be adapted to the filtration if the images of the basis elements in $V^{d}-V^{d-1}$ (resp. $V^{d}-V^{d+1}$ ) form a basis for the filtered quotient $V^{d} / V^{d-1}$ (resp. $V^{d} / V^{d+1}$ ). A basis of $V^{*}$ is said to be adapted to the dual filtrations if it is adapted to the filtration on $V^{*}$, and the dual basis elements (images of the basis elements under the isomorphism $V^{*} \rightarrow V$ ) form a basis adapted to the dual filtration of $V$.

In the cases of $\mathscr{F}_{\mathcal{L}} \mathscr{Z}^{*}$ and $\mathscr{F} \mathscr{Z}$, or $\mathfrak{D} \mathfrak{g}$ and $\mathfrak{n} \mathfrak{f} z$, equipped with the dual depth filtrations and the isomorphisms that descend from the top level of diagram (1), we say that a basis adapted to the dual filtrations is dual-depth adapted.

Lemma 2.6. Let $V$ be a finite dimensional Q-vector space $V$ equipped with an increasing filtration $\{0\}=V^{1} \subset \cdots \subset V^{d} \subset V^{d+1} \subset \cdots$ Equip the dual space $V^{*}$ with the dual decreasing filtration $\left(V^{*}\right)^{1}=V^{*} \supset \cdots \supset\left(V^{*}\right)^{d} \supset$ $\left(V^{*}\right)^{d+1} \supset \cdots$ such that $\left(V^{*}\right)^{d}$ annihilates $V^{d-1}$. Let $\delta: V^{*} \stackrel{\sim}{\rightarrow} V$ be an explicit isomorphism having the property that $\delta\left(\left(V^{*}\right)^{d+1}\right) \cap V^{d}=\{0\}$ for each $d \geq 1$. Then for every $d \geq 1$ and every $g \in\left(V^{*}\right)^{d}-\left(V^{*}\right)^{d+1}$, there exists a unique $g^{\prime} \in\left(V^{*}\right)^{d+1}$ such that $\delta\left(g+g^{\prime}\right) \in V^{d}$.

Proof. Let $g \in\left(V^{*}\right)^{d}-\left(V^{*}\right)^{d+1}$. We want to show the existence of a unique $g^{\prime} \in\left(V^{*}\right)^{d+1}$ such that $\delta\left(g+g^{\prime}\right) \in V^{d}$. Note that by hypothesis on $\delta$, the injection $\delta:\left(V^{*}\right)^{d+1} \rightarrow V$ induces an injection $\bar{\delta}:\left(V^{*}\right)^{d+1} \rightarrow V / V^{d}$ which is an isomorphism, considering the dimensions. Thus, there exists a 
unique $g^{\prime} \in\left(V^{*}\right)^{d+1}$ such that $\bar{\delta}\left(g^{\prime}\right)$ is equal to the image of $-\delta(g)$ in the quotient $V / V^{d}$. In other words, $\delta(g)+\delta\left(g^{\prime}\right) \in V^{d}$.

Lemma 2.7. Let $\delta: V^{*} \stackrel{\sim}{\rightarrow} V$ be as in Lemma 2.6. Then there exists a basis adapted to the dual filtrations for $V^{*}$.

Proof. Let $m=\operatorname{dim} V^{*}$ and let $h_{1}, \ldots, h_{m}$ be a basis adapted to the filtration for $V$, i.e. a basis such that the elements $h_{i} \in\left(V^{*}\right)^{d}-\left(V^{*}\right)^{d+1}$ pass to a basis of $\left(V^{*}\right)^{d} /\left(V^{*}\right)^{d+1}$. Correct each $h_{i}$ by an $h_{i}^{\prime}$ as in Lemma 2.6, and let $g_{1}, \ldots, g_{m}$ denote the corrected elements. Then $\delta\left(g_{1}\right), \ldots, \delta\left(g_{m}\right)$ form a basis for $V$. Let us show that it is depth adapted for the filtration on $V$. By definition of the correction, if $g_{i} \in\left(V^{*}\right)^{d}-\left(V^{*}\right)^{d+1}$, then $\delta\left(g_{i}\right) \in V^{d}$. Furthermore, the set of $g_{i} \in\left(V^{*}\right)^{d}-\left(V^{*}\right)^{d+1}$ passes to a basis for $\left(V^{*}\right)^{d} /\left(V^{*}\right)^{d+1}$, which is isomorphic to $V^{d} / V^{d-1}$, so the $\delta\left(g_{i}\right)$ for these $g_{i}$ pass to a basis of $V^{d} / V^{d-1}$. Thus the basis $g_{1}, \ldots, g_{m}$ of $V^{*}$ is adapted to the dual filtration.

Lemma 2.8. Let $\delta: V^{*} \stackrel{\sim}{\rightarrow} V$ be as in Lemma 2.6. Let $V_{d}^{*}$ denote the subspace of elements $g \in\left(V^{*}\right)^{d}-\left(V^{*}\right)^{d+1}$ such that $\delta(g) \in V^{d}$. Then $V^{*}=$ $\oplus_{d} V_{d}^{*}$, and the bases of $V^{*}$ respecting this direct sum decomposition are exactly the bases of $V^{*}$ adapted to the dual filtrations.

Proof. Let $g_{1}, \ldots, g_{m}$ be a basis for $V^{*}$ adapted to the dual filtrations. Let $\left(V^{*}\right)_{d}$ denote the subspace spanned by the $g_{i} \in\left(V^{*}\right)^{d}-\left(V^{*}\right)^{d+1}$. We claim that $\left(V^{*}\right)_{d}$ is the subspace of elements $h \in\left(V^{*}\right)^{d}-\left(V^{*}\right)^{d+1}$ such that $\delta(h) \in V^{d}$. Indeed, because the basis is adapted to the filtration, $h=\sum_{i>p} a_{i} g_{i}$, where $g_{1}, \ldots, g_{p}$ are the basis elements of level $<d$ for the filtration. But then $\delta(h)=\sum_{i>p} a_{i} \delta\left(g_{i}\right)$ and because the basis is adapted to the dual filtrations, this element lies in $V^{d}$ if and only if $a_{j}=0$ for all $g_{i} \in\left(V^{*}\right)^{d+1}$. Thus any basis adapted to the dual filtrations respects the direct sum decomposition, and conversely, any basis respecting the decomposition is obviously adapted to the dual filtrations.

Lemma 2.9. Let $\delta: V^{*} \stackrel{\sim}{\rightarrow} V$ and the spaces $\left(V^{*}\right)_{d}$ be as in Lemma 2.8 . Then $\left(V^{*}\right)_{d} \simeq\left(V^{*}\right)^{d} /\left(V^{*}\right)^{d+1}$ for $d \geq 1$.

Proof. We saw in Lemma 2.8 that if $g_{1}, \ldots, g_{m}$ is a basis adapted to the dual filtrations, then the $g_{i} \in\left(V^{*}\right)^{d}-\left(V^{*}\right)^{d+1}$ form a basis for $\left(V^{*}\right)_{d}$. Since in particular they form a basis adapted to the filtration, they also pass to a basis of $\left(V^{*}\right)^{d} /\left(V^{*}\right)^{d+1}$, yielding the desired isomorphism.

Proof of Theorem 2.2. For each $n \geq 3$, set $V^{*}=\mathfrak{D} \mathfrak{s}_{n}, V=\mathfrak{n} \mathfrak{f}_{n}$, and $\delta$ the map in (3). Let us show that $\delta$ satisfies the property of Lemma 2.6, i.e. that $\delta\left(\mathcal{D} \mathfrak{S}_{n}^{d+1}\right) \cap \mathfrak{n} \mathfrak{f}_{n}^{d}=\{0\}$ for $d \geq 1$.

Let $f \in \mathcal{D}_{n}^{d+1}$ and write $f=\sum_{w} a_{w} w$ over words $w$ all having at least $d+1 y$ 's, and suppose that $\delta(f) \in \mathfrak{n} \mathfrak{F}_{n}^{d}$. Then by definition of the dual 
filtrations, which are the depth filtrations in this situation, the scalar product $(f \mid \delta(f))=0$. But if we consider $f \in \mathrm{Q}\langle x, y\rangle$, then we can consider $\delta(f) \in \mathrm{Q}[\bar{Z}(w)]$, and the value of the scalar product of $f$ and $\delta(f)$ is the same, namely

$$
(f \mid \delta(f))=\sum_{w} a_{w}^{2} .
$$

Thus it is equal to zero if and only if $f=0$, showing that $\delta$ satisfies the hypothesis of Lemma 2.6.

Then Lemma 2.7 shows that dual-depth adapted basis exist. From Lemma 2.9, we deduce that the direct-sum decomposition exists, that the dualdepth adapted bases are in bijection with the bases that respect it, and finally, the isomorphism with the associated graded. This concludes the proof of Theorem 2.2.

\section{Canonical ring generators for the formal multiple zeta value algebra}

By the duality isomorphism $\delta$, any explicit basis of $\delta \xi$ yields not only a basis of $\mathfrak{n} \mathfrak{f}_{z}$, but by considering $\mathfrak{D} \mathfrak{S} \subset \mathscr{F}_{\mathscr{Z}^{*}}$, also a set of irreducibles (ring generators) for $\mathscr{F} \mathscr{Z}$. Thus, a dual-depth adapted basis for $\mathfrak{D} \mathfrak{g}$ yields a set of irreducibles, and in particular, for any values of $n$ and $d$ such that $\operatorname{dim} D \mathfrak{S}_{n}^{d} / \mathcal{D} \mathfrak{S}_{n}^{d+1}=1$, we obtain a canonical irreducible (up to scalar multiple).

No dimensions larger than 1 appear in the associated graded for weights $3 \leq n \leq 12$; thus the dual-depth adapted bases for $\delta \mathfrak{S}_{n}$ for these values of $n$ are uniquely determined (up to scalar multiple), providing canonical irreducibles in those weights.

For the depth 1 irreducibles that occur for odd values of $n$, we fix the scalar multiple by choosing the unique $f_{n} \in \mathfrak{D} \mathfrak{S}_{n}$ satisfying $\delta\left(f_{n}\right)=z(n) \in \mathfrak{n} \mathfrak{\mathfrak { T }} \mathfrak{z}_{n}$ for $n=3,5,7,9,11$. For example, $f_{3}=\frac{1}{12}([x,[x, y]]+[[x, y], y])$. The first main result of this paper is the singling out of these canonical depth 1 irreducibles in odd weight, which conjecturally form a set of generators for the Lie algebra $D S$.

The second main result is the production of irreducibles in higher depth, by taking dual-depth adapted bases of $D \mathfrak{g}_{n}$ for each $n$, but the irreducibles obtained this way are not uniquely determined except for those of depths $d$ where $\operatorname{dim} \mathfrak{D} \mathfrak{S}_{n}^{d} / \mathfrak{D} \mathfrak{S}_{n}^{d+1}=1$. Since $\operatorname{dim} \mathfrak{D} \mathfrak{s}_{12}^{4} / \mathfrak{D} \mathfrak{S}_{12}^{5}=1$, our method does produce two canonical (up to scalar multiple) irreducible generators of weight 12 in $\mathscr{F}_{\mathscr{Z}}{ }_{12}$. Such explicit canonical generators have not been found previously in any depth, even $d=1$ (though J. Ecalle has proposed several alternative methods to find bases, cf. [1]). 
We give the dual-depth adapted bases of $\mathfrak{D} \mathfrak{S}_{n}$ up to weight $n=12$ in the following table, where the $f_{n}$ denote the canonical elements defined by $\delta\left(f_{n}\right)=\zeta(n) \in \mathfrak{n} \mathfrak{f}_{z_{n}}$.

DUAL-DEPTH ADAPTED BASES UP TO WEIGHT 12

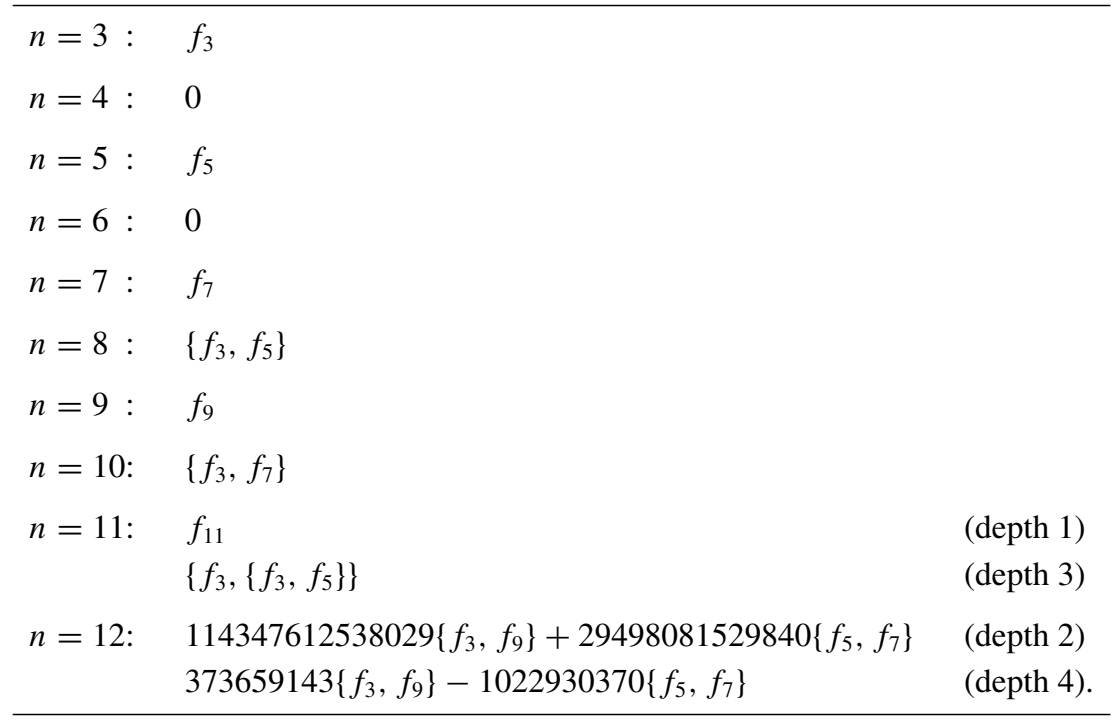

The next table translates these bases into canonical irreducible generators for $\mathscr{F} \mathscr{Z}$. This calculation is done based on the diagram (1). The Lie algebra $\mathfrak{D} \mathfrak{S}$ is contained in the Hopf algebra $\mathscr{F}_{\mathscr{Z}} \mathscr{Z}^{*} \subset \mathrm{Q}\langle x, y\rangle$, so elements of $\mathfrak{D} \mathfrak{S}$ are nothing but polynomials which lie in $\mathscr{F}^{*}$. If $f=\sum_{w} a_{w} w$ is an element of $\mathrm{D} \mathfrak{S}$, we can thus consider it in $\mathscr{F} \mathscr{Z}^{*}$, and its dual is simply given by $\delta(f)=\sum_{w} a_{w} Z(w) \in$ $\mathscr{F} \mathscr{Z}$. To simplify the final expressions, we have fixed a basis of $\mathscr{F}_{\mathscr{Z}}$ in each weight $3 \leq n \leq 12$ (the chosen basis contains irreducible multizeta values and products), and used a computer program written in Maple to express each $Z(w)$ in this basis to obtain a simpler expression for $\delta(f)$.

DUAL-DEPTH ADAPTED IRREDUCIBLES OF $\mathscr{F} \mathscr{Z}$ UP TO WEIGHT 12

$$
\begin{array}{ll}
\mathscr{F}_{\mathscr{Z}_{3}^{1}}^{1} & Z(3) \\
\mathscr{F}_{\mathscr{Z}_{5}^{1}}^{1} & Z(5)-\frac{3}{11} Z(2) Z(3) \\
\mathscr{F}_{\mathscr{Z}_{7}^{1}}^{1} & Z(7)-\frac{61}{213} Z(2) Z(5)-\frac{226}{2343} Z(3) Z(2)^{2} \\
\mathscr{F}_{\mathscr{Z}_{8}^{2}}^{2} & -\frac{24453}{2} Z(6,2)-\frac{60027}{10} Z(2)^{4}+\frac{77649}{2} Z(3) Z(5)-1683 Z(2) Z(3)^{2}
\end{array}
$$




$$
\begin{aligned}
& \mathscr{F}_{\mathscr{Z}_{9}^{1}}^{1} \quad Z(9)-\frac{1242768}{13065005} Z(2)^{2} Z(5)-\frac{3697886}{91455035} Z(2)^{3} Z(3)-\frac{1479309}{5226002} Z(2) Z(7) \\
& +\frac{50274}{2613001} Z(3)^{3} \\
& \mathscr{F}_{\mathscr{Z}_{10}^{2}} \quad-\frac{6614309}{32} Z(8,2)+\frac{7569029}{16} Z(5)^{2}-\frac{5656181999}{61600} Z(2)^{5}-\frac{703339}{4} Z(5) Z(2) Z(3) \\
& +\frac{7205263}{8} Z(3) Z(7)+\frac{84201}{2} Z(2) Z(6,2)-\frac{2698111}{80} Z(2)^{2} Z(3)^{2} \\
& \mathscr{F}_{11}^{1} \quad Z(11)-\frac{89344789701892}{4831579726916775} Z(2)^{4} Z(3)-\frac{1139040}{28492287937} Z(3) Z(6,2) \\
& -\frac{71379583588}{256430591433} Z(2) Z(9)+\frac{30559167920}{541353470803} Z(5) Z(3)^{2}-\frac{3101026096}{541353470803} Z(2) Z(3)^{3} \\
& -\frac{47806388544}{541353470803} Z(2)^{2} Z(7)-\frac{143810356328}{3789474295621} Z(5) Z(2)^{3} \\
& \mathscr{F}_{11}^{3} \quad-1841904 Z(8,2,1)-\frac{425314163}{4} Z(11)+\frac{104438821}{140} Z(2)^{4} Z(3) \\
& -1333368 Z(3) Z(6,2)+\frac{204483287}{6} Z(2) Z(9)+\frac{3279105}{2} Z(5) Z(3)^{2} \\
& -78972 Z(2) Z(3)^{3}+\frac{36753903}{4} Z(2)^{2} Z(7)+\frac{124168102}{35} Z(5) Z(2)^{3} \\
& \mathscr{F}_{\mathscr{Z}_{12}^{2}}^{2}-\frac{195054138110588251}{64} Z(10,2)-\frac{8653643469032603353873}{7644000} Z(2)^{6} \\
& +\frac{1077831097314738125}{64} Z(3) Z(9)+\frac{1161844161287230993}{64} Z(5) Z(7) \\
& -\frac{110785136392182147}{64} Z(2) Z(5)^{2}-\frac{194318216436736119}{64} Z(7) Z(2) Z(3) \\
& -\frac{591899366895484531}{1680} Z(2)^{3} Z(3)^{2}-\frac{266292350528747767}{160} Z(5) Z(3) Z(2)^{2} \\
& +\frac{32687838480401691}{160} Z(2)^{2} Z(6,2)+\frac{81857357214535231}{128} Z(2) Z(8,2) \\
& +\frac{1256483182613535}{16} Z(3)^{4} \\
& \mathscr{F}_{\mathscr{Z}_{12}^{4}}^{4} \quad-\frac{2088509495}{48} Z(8,2,1,1)+\frac{40513131683}{256} Z(10,2)-\frac{12283082576753783}{168168000} Z(2)^{6} \\
& +\frac{328220974925}{384} Z(3) Z(9)+\frac{1782213740011}{1536} Z(5) Z(7)-\frac{69224488487}{768} Z(2) Z(5)^{2}
\end{aligned}
$$




$$
\begin{aligned}
& -\frac{19970763059}{128} Z(7) Z(2) Z(3)-\frac{553292096341}{20160} Z(2)^{3} Z(3)^{2} \\
& -\frac{131370683233}{2880} Z(5) Z(3) Z(2)^{2}-\frac{48581102573}{1440} Z(2)^{2} Z(6,2) \\
& -\frac{454799848867}{4608} Z(2) Z(8,2)+\frac{2024205035}{576} Z(3)^{4}
\end{aligned}
$$

\section{REFERENCES}

1. Ecalle, J.,ARI/GARI, la dimorphie et l'arithmétique des multizêtas: un premier bilan, J. Théor. Nombres Bordeaux 15 (2003), 411-478.

2. Furusho, H., The multiple zeta value algebra and the stable derivation algebra, Publ. Res. Inst. Math. Sci. 39 (2003), 695-720.

3. Furusho, H.,Double shuffle relation for associators, Ann. of Math. 174 (2011), 341-360.

4. Goncharov, A.,Galois symmetries of fundamental groupoids and noncommutative geometry, Duke Math. J. 128 (2005), 209-284.

5. Racinet, G.,Doubles mélanges de polylogarithmes multiples aux racines de l'unité, Publ. Math. Inst. Hautes Études Sci. 95 (2002), 185-231.

EQUIPE ANALYSE ALGÉBRIQUE

INSTITUT DE MATHÉMATIQUES DE JUSSIEU

4 PLACE JUSSIEU, CASE 247

75252 PARIS CEDEX

FRANCE

E-mail: leila@math.jussieu.fr 